\title{
ANALYSIS AND OPTIMIZATION OF "FULL-LENGTH" DIODES*
}

\author{
by \\ Alfred Schock \\ Republic Aviation Division of Fairchild Hiller Corporation \\ Farmingdale, L. I., New York
}

\begin{abstract}
A method of analyzing the axial variation of the heat generation rate, temperature, voltage, current density and emitter heat flux in a thermionic converter is described. The method is particularly useful for the case of "long" diodes, each extending over the full length of the reactor core. For a given diode geometry and fuel distribution, the analysis combines a nuclear solution of the axial fission density proflle with the iterative solution of four differential equations representing the thermal, electrical, and thermionic interactions within the diode. The digital computer program developed to solve these equations can also perform a design optimization with respect to lead resistance, load voltage, and emitter thickness, for a specified maximum emitter temperature. Typical results are presented, and the use of this analysis for predicting the diode operating characteristics is illustrated.
\end{abstract}

\section{Introduction}

A thermionic reactor design study ${ }^{(1)}$ currently under way is based on the concept of using long, cylindrical diodes, each extending over the full length of the reactor core. In such a design, accurate knowledge of the diode's axial voltage and temperature profiles is vital, since the optimum converter length represents a compromise between minimum neutron leakage and minimum ohmic loss.

Earlier ohmic loss calculations have usually assumed a uniform emitter current density, an assumption that is clearly inaccurate since the existence of significant voltage variation must necessarily lead to a redistribution of the diode current. Similarly, thermionic system studies have frequently treated each diode as isothermal, even though the rather substantial emitter lead heat loss must result in correspondingly large temperature gradients near the end(s) of

* Work supported by U. S. Atomic Energy Commission. 
the emitter. With an axially uniform heat generation rate, this effect can be shown to lead to appreciable temperature non-uniformities, extending over a significant fraction of the total emitter length. The resultant penalty in diode power is important not only in the full-length design discussed above, but also in the shorter diodes used in conventional designs. In the case of full-length diodes, however, it is possible to compensate for the emitter's end losses and improve its temperature uniformity, by using a combination of axial reflectors and fuel zoning to produce a skewed fission profile. Thus, a detailed axial analysis is particularly important in full-length diodes.

Such an analysis must provide close coupling between the various nuclear, electrical, thermal, and thermionic equations because of their mutual interdependence, particularly the effect of local diode voltage and emitter temperature on current density and electron cooling. In the present study, the relationship between these variables was obtained from an extensive set of thermionic performance data that had been generated by the recently developed ${ }^{(2)}$ SIMCON computer code. These data extend over a wide range of emitter temperatures (1700 to $2400^{\circ} \mathrm{K}$ ), collector temperatures $\left(850\right.$ to $\left.1150^{\circ} \mathrm{K}\right)$, and cesium reservoir temperatures $\left(550\right.$ to $\left.690^{\circ} \mathrm{K}\right)$. A specialized "thermionic correlation" subroutine TICORR, capable of performing multi-dimensional interpolations, was employed to compute the local current density and emitter heat flux.

\section{Analysis}

Within each diode, a complete thermal and electrical analysis should really be three-dimensional (or two-dimensional in the case of axisymmetric converters). However, since the axial dimension of a full-length diode is much larger than the transverse dimensions, and since current flow is primarily in the axial direction, we shall simplify the problem by treating it as one-dimensional.

The first step of the analysis is to compute the axial fission density profile $f(z)$ for the assumed fuel distribution. This is done by means of the ANISN $(3)$ one-dimensional, multi-group, multi-zone neutron transport code, with transverse leakage represented by appropriate buckling terms. Beyond the actual core, additional zones are included to provide a realistic representation of axial reílectors and various diode components (e.g., power leads, seals, coolant 
plenums). The computed fission density $f(z)$ is normalized to unity at $z=0$, the reactor midplane.

To carry out the thermal and electrical analysis of the fuel-emitter structure, it is convenient to employ four simultaneous first-order differential equations, describing the variation of the axial heat flow rate $Q$ and current $I_{E}$, the emitter temperature $T$ and the local diode voltage $V$. The axial heat flow $Q$ in the fuel-emitter assembly varies because of heat generated by fissions, heat lost at the emitter surface $r=r_{\mathrm{E}}$, and ohmic heating:

$$
d Q / d z=A_{P} Q_{O}^{\prime \prime \prime} f(z)-2 \pi r_{E} q_{E}(V, T)+I_{E}^{a} / A_{E} \sigma_{\xi}^{\prime} .
$$

Here, $A_{F}$ and $A_{E}$ respectively denote the cross-sectional areas of the fuel and emitter, $Q_{0}^{\prime \prime \prime}$ is the volumetric heat generation rate in the fuel at the reactor midplane $(z=0), q_{\mathrm{E}}$ represents the emitter heat flux at the local voltage $V$ and temperature $T$, and $\sigma_{E}^{\prime}$ is the effective electrical conductivity of the emitter. The latter includes not only the actual conductivity of the emitter, but also the possible contribution of a conductive fuel. Moreover, in the case of the externalfuel design (1) where the fuel surrounds the emitter, the outer sheath may also contribute to conduction if an electrical path exists between sheath and emitter. Thus, the effective emitter conductivity $\sigma_{E}^{\prime}$ is defined in its most general form by:

$$
A_{\mathrm{E}} \sigma_{\mathrm{E}}^{\prime} \equiv A_{\mathrm{E}} \sigma_{\mathrm{E}}+A_{F} \sigma_{\mathrm{F}}+A_{\mathrm{s}} \sigma_{\mathrm{s}} .
$$

Since we shall strive for an isothermal emitter, the effect of temperature on conductivity will be ignored within the diode.

Next, the axial current $I_{E}$ in the fuel-emitter assembly varies because of electrons leaving the emitter surface. Thus

$$
d I_{\mathrm{E}} / d z=2 \pi r_{\mathrm{E}} J(V, T),
$$

where $J$ is the thermionic current density at the local diode voltage $V$ and emitter temperature $T$. The axial variation of $T$ is defined by the usual conduction equation,

$$
d T / d z=-Q / A_{\mathrm{E}} K_{\mathrm{E}}^{\prime},
$$


where the definition of the effective thermal conductivity $K_{\varepsilon}^{t}$, in its most general form, is analogous to Eq. (2):

$$
A_{E} K_{\varepsilon}^{\prime} \equiv A_{E} K_{E}+A_{F} K_{F}+A_{S} K_{S} .
$$

Finally, to derive the fourth differential equation, we note that the local diode voltage $V$ is the difference between the emitter and collector voltages,

$$
V=V_{\varepsilon}-V_{c} .
$$

These are related to the corresponding currents by Ohm's law:

$$
\begin{aligned}
& d V_{\mathrm{E}} / \partial z=-T_{E} / A_{C} \sigma_{E}^{\prime}, \\
& d V_{C} / d z=-I_{C} / A_{C} \sigma_{C} .
\end{aligned}
$$

We must now differentiate between two types of diode connections. If the emitter lead is connected to one end of the diode and the collector lead to the opposite end, the currents $T_{\varepsilon}$ and $T_{\varepsilon}$ flow in the same direction, and their sum equals the diode current $I_{0}$ :

$$
I_{E}+I_{c}=I_{0} \text {. }
$$

In that case the diode voltage variation, obtained by combining Eqs. $(6),(7),(8)$, and (9a), is given by:

$$
d V / d z=-I_{E} / A_{E} \sigma_{E}^{\prime}+\left(I_{D}-T_{E}\right) / A_{C} \sigma_{C} \text {. }
$$

If, on the other hand, the emitter and collector leads are connected to the same end of the diode, then the currents $I_{\mathrm{E}}$ and $I_{\mathrm{c}}$ are of opposite direction and equal magnitude:

$$
I_{E}+I_{c}=0
$$

In that case, combining Eqs. (6), (7), (8) and (9b) yields:

$$
d V / d z=-\left[\left(A_{E} \sigma_{e}^{\prime}\right)^{-1}+\left(A_{c} \sigma_{c}\right)^{-1}\right] I_{E} .
$$

The present study assumes the use of double-ended diodes, with a set of emitter and collector leads at each end of the converter. This arrangement minimizes ohmic losses, since such a converter is electrically equivalent to two single-ended diodes of half the length. Equation (10b) therefore constitutes our fourth differential equation. 


\section{Boundary Conditions}

The system of Eqs. (1), (3), (4), and (10b) requires a total of four boundary conditions. Because of symmetry about the reactor midplane, we conclude that the axial heat flow $Q$ and current $I_{\mathrm{E}}$ are each zero at $z=0$. To define the other two boundary conditions, we must first relate the net diode voltage $V_{D}$ to the heat flow $Q_{1}$, current $I_{1}$, voltage $V_{1}$, and emitter temperature $T_{1}$ at $z=z_{1}$, the junction of the emitter and the emitter lead.

In general, the lead may have a non-uniform cross-sectional area $A_{6}(z)$, and its cold junction operates at the collector temperature $I_{c}$. Moreover, since $T_{1}$ and $T_{c}$ differ considerably, the temperature dependence of its electrical and thermal conductivities should not be ignored. For an emitter lead passing a current $I_{1}$, the temperature and heat flow gradients (neglecting radiation) are given by

$$
d T / d z=-Q / A_{\mathrm{l}} K_{\mathrm{l}}(T) \text {, }
$$

and

$$
d Q / d z=I_{1}^{2} / A_{L} \sigma_{L}(T)
$$

The emitter lead conductivities $K_{L}$ and $\sigma_{L}$ are assumed to obey the WiedemannFranz law,

$$
K_{L} / C_{L}=\frac{1}{3}(\pi / k / e)^{2} T,
$$

where $k$ is Boltzmann's constant and $e$ the electronic charge. Dividing Eq. (12) by (11) and combining the result with (13), we obtain the separable equation,

$$
d Q / d T=-\frac{1}{3}\left(\pi k I_{1} / e\right)^{2}(T / Q) .
$$

Since the emitter lead neither consumes nor produces energy, it must satisfy the energy balance

$$
I_{1} V_{1}+Q_{1}=I_{1} V_{6}+Q_{\mathrm{C}} \text {, }
$$

where $Q_{c}$ is the heat flow from lead to collector.

Integrating Eq. (14) from $T=T_{1}, Q=Q_{1}$ to $T=T_{C}, Q_{\mathrm{C}}=Q_{\mathrm{c}}$ and solving for the net diode voltage, we obtain:

$$
V_{0}=V_{1}-\left[\left(Q_{1} / I_{1}\right)^{2}+\frac{1}{3}(\pi k / e)^{2}\left(T_{1}^{2}-T_{c}^{2}\right)\right]^{\frac{1}{2}}
$$


Finally, the emitter lead resistance $R_{L}$ must, of course, obey Ohm's law:

$$
R_{L}=\left(V_{1}-V_{D}\right) / I_{2}
$$

Equations (16) and (17) constitute the required third and fourth boundary conditions. Thus, for a diode of given geometry and fission density profile $f(z)$, the operating point is completely defined, at least in principle, by specifying the central heat generation rate $Q_{0}$, lead resistance $R_{L}$, and a single parameter to characterize the load, such as the voltage $V_{0}$, current $I_{1}$, or resistance $V_{0} / I_{1}$. In practice, however, solutions can only be found for a limited range of parameters, because of the limited extent of the available SIMCON ${ }^{(2)}$ data needed by the TICORR subroutine to compute the emitter current density $J(V, T)$ in Eq. (3) and the emitter heat flux $q_{\mathrm{E}}(V, T)$ in Eq. (1).

\section{Computer Program}

In the course of the present study, a digital computer program TET(thermal, electrical, thermionic) was developed for solving the set of four differential equations. Since two of the required boundary conditions apply to the reactor midplane $(z=0)$ and the other two to the emitter-lead junction $\left(z=z_{1}\right)$, a two-level trial and error procedure must be employed; e.g., by guessing at the midplane voltage $V_{0}$ and emitter temperature $T_{0}$. Each trial requires numerical integration of the four equations, with the use of TICORR for thermionic data interpolation at each mesh point. The trials are repeated until boundary conditions 3 and 4 are satisfied,

In actual practice, rather than specify the heat generation rate $80^{\mathrm{N \prime}}$ and solve for the central emitter temperature $\tau_{0}$ by trial and error, it was found more efficient to solve for $Q_{0}^{\prime \prime \prime}$ after first specifying $T_{0}$ (which is usually, though not always, the maximum emitter temperature).

In addition to finding solutions for specified values of the emitter temperature $T_{0}$, lead resistance $R_{L}$, and load voltage $V_{0}$, the TET program also provides the option of optimizing the leads and load, i.e., finding the values of $B_{L}$ and $V_{0}$ which maximize the net diode power $F_{0}=2 I_{1} V_{0}$. Moreover, if desired TET can also determine the optimum value of the emitter cross-sectional area $A_{E}$, i.e. the value which maximizes the diode's volumetric power density,

$$
P_{0}^{\prime \prime \prime}=I_{1} V_{D} /\left(\pi r_{E}^{2}+A_{E}\right) z_{1} .
$$


Finally, the TET program also solves for the net conversion efficiency,

$$
\eta=I_{1} V_{0} / A_{\mathrm{F}} Q_{0}^{\prime \prime \prime} \int_{0}^{z_{1}} f^{\prime}(z) d z \text {. }
$$

\section{Results and Conclusions}

A large number of TET optimizations were carried out, covering a range of emitter diameters $(1.25$ to $2.5 \mathrm{~cm})$, diode lengths $(20$ to $25 \mathrm{~cm})$, and fuel distributions (flat and zoned). The problems generally postulated a maximum emitter temperature of $2073^{\circ} \mathrm{K}$, a collector temperature of $1000^{\circ} \mathrm{K}$, a reservoir temperature of $630^{\circ} \mathrm{K}$, and a $0.025 \mathrm{~cm}$ electrode spacing.

We have assumed tungsten emitters and sheaths, with bulk $\mathrm{UO}_{2}$ fuel wafers (normal to the diode axis), separated by tungsten fins occupying from 15 to $50 \%$ of the fuel chamber volume. These fins serve to reduce the maximum fuel temperature, and their thickness can be varied to adjust the axial fission density profile. The fuel chamber itself was generally assumed to occupy $40 \%$ of the total core volume. Actually, the results are virtually independent of this assumption, since $\mathrm{UO}_{2}$ has no electrical conductivity and very little thermal conductivity. In the case of a conductive cermet fuel, however, the fuel volume fraction would be an important parameter.

\section{Design Optimization}

The emitter lead resistance was found to have a very broad optimum. As illustrated by Figure 1, the lead resistance can be varied over a surprisingly wide range without incurring excessive performance penalties. This is fortunate, since it permits structural and mechanical considerations to influence the lead design. The high currents generated in full-length diodes favor the use of very thick leads.

The net diode power was found to be a far more sensitive function of the diode voltage $V_{0}$, as illustrated by Figure 2 (for optimized lead resistance). It was found that the optimum load voltage is essentially independent of the emitter's diameter, length, or cross-sectional area; it does depend on cesium pressure and maximum emitter temperature. 
The effect of varying the emitter's cross-sectional area is illustrated by Figure 3 , for optimized lead and load conditions. The figure also illustrates the weak effect of collector temprrature, with $1000^{\circ} \mathrm{K}$ giving the best results. The emitter thickness was consistently found to optimize at rather low values, in spite of the fact that the se correspond to high ohmic losses. However, the optimum is seen to be quite broad, permitting the choice of thicker emitters to reduce ohmic losses and enhance the diode's structural integrity. Thus, the use of a $0.25 \mathrm{~cm}-$ thick emitter only results in a relatively small penalty in net power density.

Figure 4 shows the effect of emitter diameter and length on the diode's net volumetric: power density, with optimized lead resistance, load voltage, and emitter thickness. Over the range investigated, the power density increases monotonically with decreasing emitter diameter, which should therefore be sized at the lowest value consistent with the requirement for structural integrity and dimensional stability.

Similarly, the volumetric diode power density can be increased by reducing the diode length, because of reduced ohmic loss. However, this length reduction increases the axial neutron leakage, and therefore raises the fuel volume required to maintain criticality. Thus, while there is no optimum length from the viewpoint of diode power density, there is an optimum with respect to the reactor power density. Extensive design calculations ${ }^{(1)}$ revealed this to be about 20 to $25 \mathrm{~cm}$, over a very wide range of reactor power levels.

Finally, Figures 5 and 6 display detailed axial solutions for two identical diodes, to illustrate the effect of fuel zoning. Figure 5 assumes a uniform fuel, with UO $\mathrm{O}_{2}$ occupying $85 \%$ of the fuel chamber volume. In the second case, as shown by Figure 6 , the fuel is divided into 12 axial zones, with an average $\mathrm{UO}_{2}$ content in the fuel chamber of $66 \%$ (varying from $50 \%$ in the center to $85 \%$ near the ends).

Both cases assume the same axial reflectors, including seals, power leads, coulant plenum, and $10 \mathrm{~cm}$ of $\mathrm{BeO}$. The resultant fission density profiles, as computed by ANISN ${ }^{(3)}$, are shown in Figures 5 and 6 . The remaining curves (showing the axial variation of emitter temperature, current density, voltage, and power density) are self-explanatory. The principal data about the two cases are summarized in Table $\mathrm{I}$. 
As can be seen, a flat fuel distribution leads to a highly non-uniform temperature profile, primarily because of heat losses to the emitter leads. Since converter performance is very sensitive to emitter temperature, the last $20 \%$ of the diode is relatively ineffective.

It should be noted that this problem also applies to conventional, short diodes. It can be shown that the heat lost to the emitter leads (expressed as a fraction of total heat generation) and the extent of the resultant temperature depression (expressed as a fraction of total diode length) are essentially the same for short and long diodes. In the latter case, however, considerable improvement can be achieved by axial fuel zoning.

The particular zoning illustrated in Figure 6 is not necessarily optimum; other calculations have shown that almost the same improvement can be achieved using just three coarse zones. Note, however, that the goal should be to produce a skewed rather than a flat fission profile, to compensate for heat losses at the ends of the diode. With such a fission profile it is seen that the emitter temperature can be maintained near its maximum value over a much larger fraction of the total diode length.

As shown by the overall performance summary in Table $I$, the use of fuel zoning raises the optimum diode voltage from 0.531 to 0.601 volt, the net diode power from 930 to 1154 watts, and the net conversion efficiency from 12,7 to 13. $9 \%$. However, it must be kept in mind that this improvement is only achieved at the price of lowering the $\mathrm{UO}_{2}$ volume fraction in the fuel chamber from $85 \%$ to $66 \%$. Thus, axial fuel zoning makes it possible to achieve a given reactor power output with fewer diodes, but with each diode having a larger fuel chamber. Consequently, the overall effect of zoning on critical core size must be determined for each specific case, and cannot be predicted a priori.

\section{Diode Characteristics}

Thus far our discussion has dealt with the use of the present analysis to optimize the diode dimensions and choose the best operating point. However, the same analytical program (without the optimization options) can also be used to determine the current-voltage characteristics for a diode of fixed design. Figure 7 presents such a set of characteristics, for the same diode design represented in 
Figure 6. The solid curves are for constant thermal power of the reactor, while the dashed lines are for constant maximum emitter temperature. The thermal power is expressed in relative terms, where unity represents the heat generation rate ( 8300 watts per diode) of the operating point illustrated by Figure 6 .

The curves shown in Figure 7 are of practical value not only for designing the reactor and its control system, but also for assessing the electrical effect of open-circuit failures on total reactor power. To minimize the effect of open circuits (including loss of cesium) is is desirable to connect diodes in parallel groups, before connecting these groups in series. In that way, when a particular diode is open-circuited its group-partners can make up the current deficit, although at the penalty of operating at a lower (possibly even negative) output voltage.

The significant fact illustrated by Figure 7 is that this voltage penalty is much greater in the case of constant thermal power input than would be predicted from the constant temperature diode characteristics usually shown. This is so because raising the diode current results in increased electron cooling; at constant heat input this lowers the emitter temperature.

For example, for the design point depicted in Figure 6 and represented by point $P$ in Figure 7 , increasing the diode current by $45 \%$ above its design value lowers the maximum emitter temperature by $215^{\circ} \mathrm{K}$ and reduces the output voltage to zero. It appears questionable whether very substantial current increases beyond this point could be achieved, because of the rapidly decreasing emitter temperature. Since any reliability analysis must contemplate the occurrence of more than one open-circuited diode per parallel group, this places a lower limit on the number of diodes per group to ensure continued system operation in spite of such multiple failures. A detailed discussion of this point is beyond the scope of the present paper.

Finally, it was found that under certain conditions the TET equations and boundary conditions may be satisfied by more than one axial solution. This condition arises because the emitter current density and heat flux can actually diminish at higher temperatures, as a result of cesium desorption. The phenomenon is illustrated by Figure 8 for the same diode design, heat input, and fission profile 
represented by Figure 6 , but with a $620^{\circ} \mathrm{K}$ cesium reservoir and a 0,535 -volt load. The axial variation of temperature, voltage, and current density for the high and low cesium coverage solutions is represented in Figure 8 by the dashed and solid curves, respectively. Comparison of the two solutions shows that Case II has a $260^{\circ} \mathrm{K}$ higher maximum emitter temperature, and a $16 \%$ lower output current.

This co-existence of more than one possible operating point is similar to a phenomenon in the case of isothermal, isopotential diodes. By analogy, we infer that still a third solution of the TET equations exists, but at extremely high temperatures, beyond the range of the SIMCON data. A detailed discussion of this phenomenon, which can have considerable practical significance, is presented in another paper. ${ }^{(4)}$

\section{Acknowledgments:}

The author wishes to express his appreciation for valuable help given by C. L. Eisen and M. J. Abbate, and for the SIMCON-generated thermionic performance data tabulations furnished by D. R. Wilkins.

\section{References:}

(1) M. J. ABBATE, C. L. EISEN, B. RAAB, and A. SCHOCK, "ExternalFuel Thermionic Reactors," Proceedings of the Second International Conference on Thermionic Electrical Power Generation, May 1968.

(2) D. R. WILKINS, "An Improved Theoretical Description of Thermionic Converter Performance Characteristics, "Report on Twenty-Seventh Annual Physical Electronics Conference," M.I. T., Cambridge, Mass., March 1967.

(3) W. W. ENGLE, Jr., "A. Users Manual for ANISN, A One Dimensional Discrete Ordinates Transport Code with Anisotropic Scattering," United Carbide Corporation, Nuclear Div., Report K-1693, March 30, 1967.
A. SCHOCK, "Effect of Cesium Pressure on Thermionic Stability," Proceedings of the Second International Conference on Thermionic Electrical Power Generation, May 1968. 
TABLE I: Effect of Fuel Zoning on Diode Performance

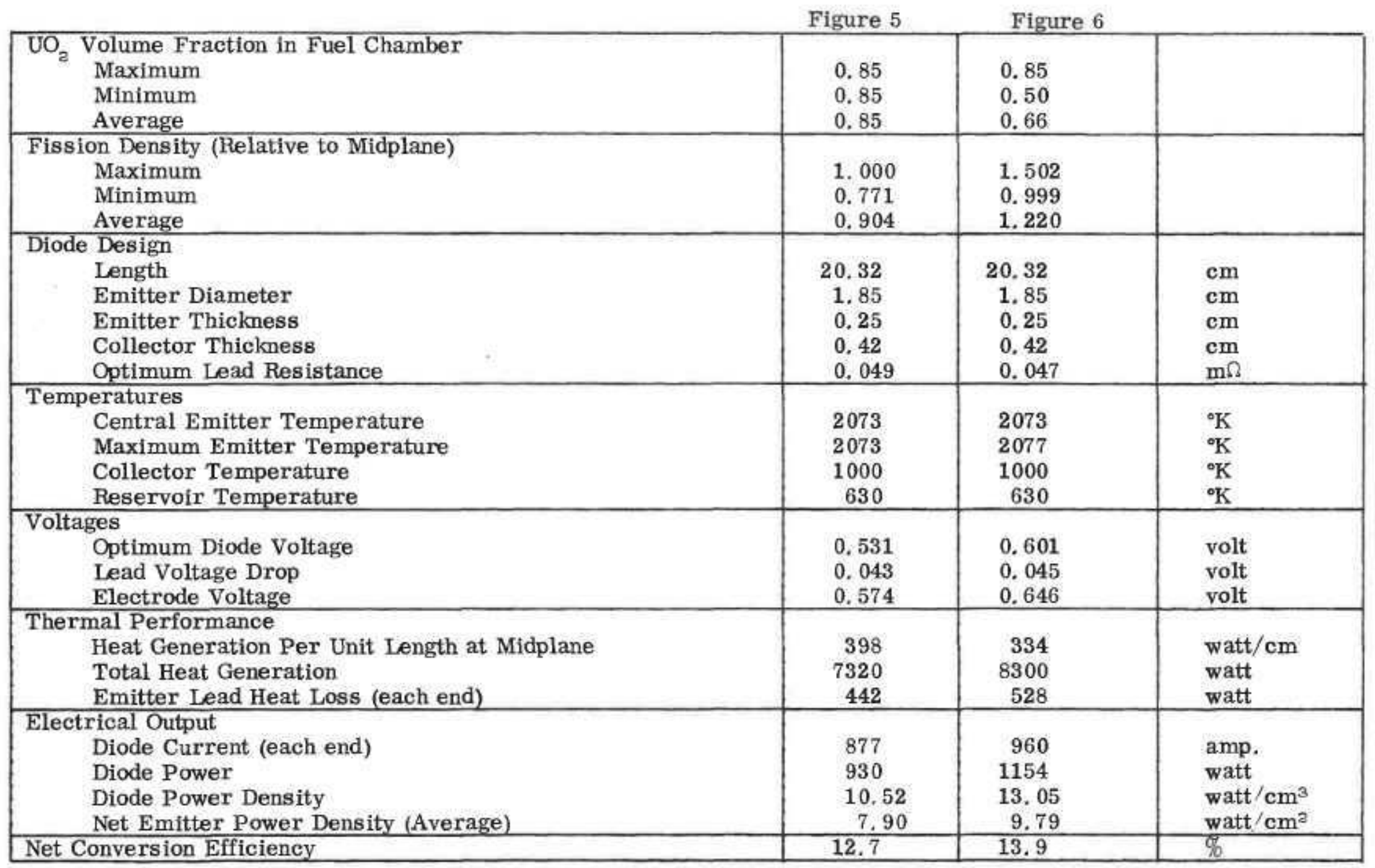


Fig. 1: Effect of Lead Resistance

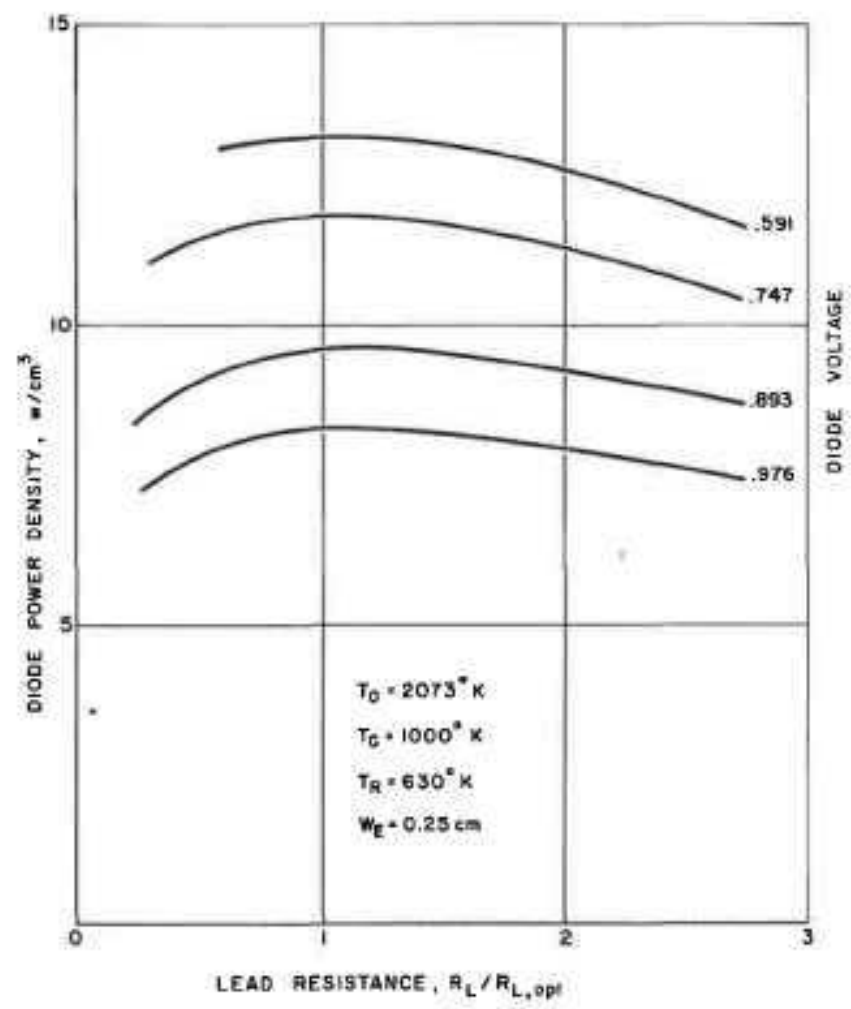

Fig. 3: Effect of Emitter Thickness

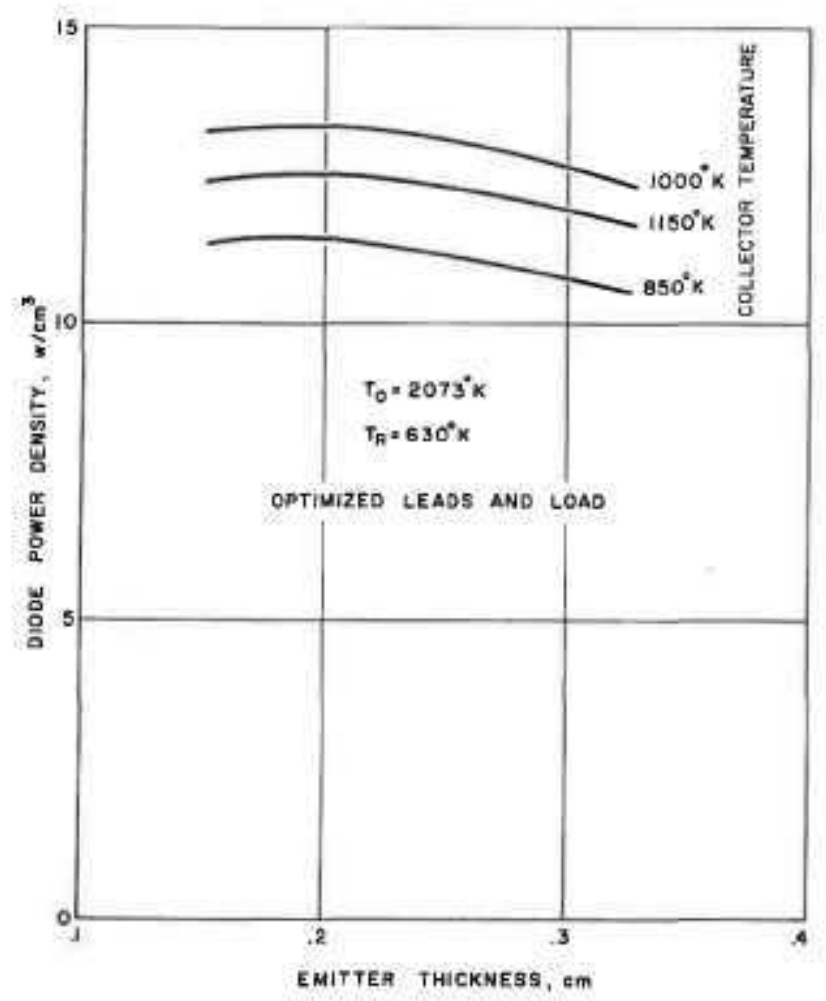

Fig. 2: Effect of Diode Voltage

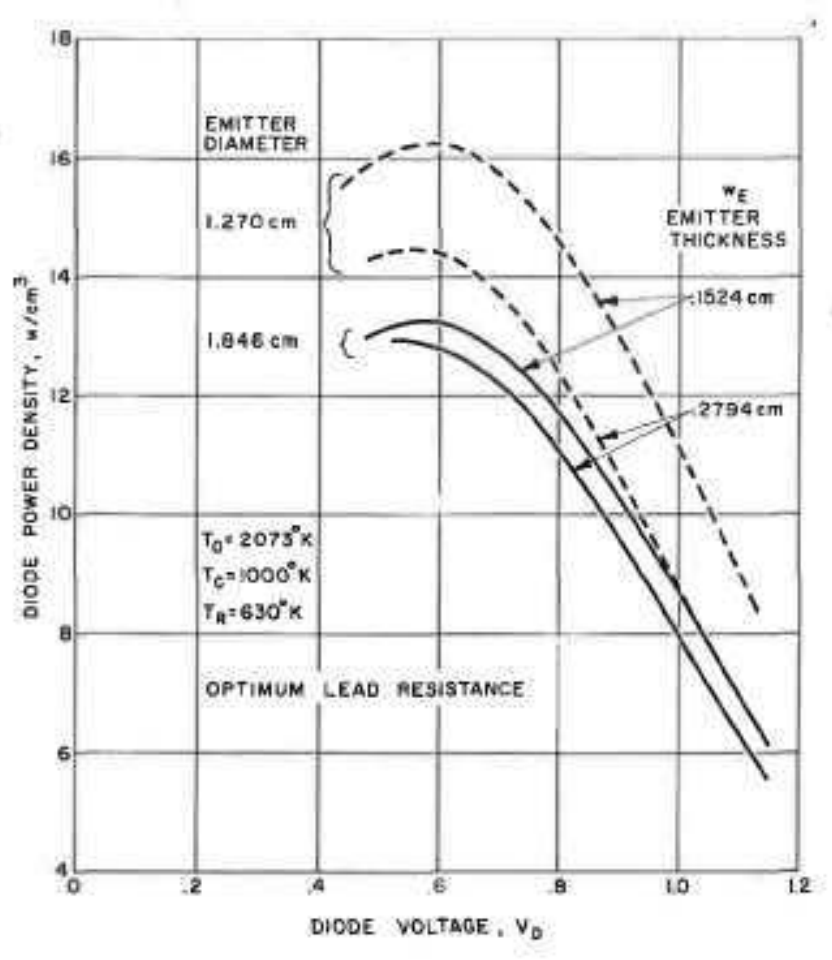

Fig. 4: Effect of Emitter Diameter and Length

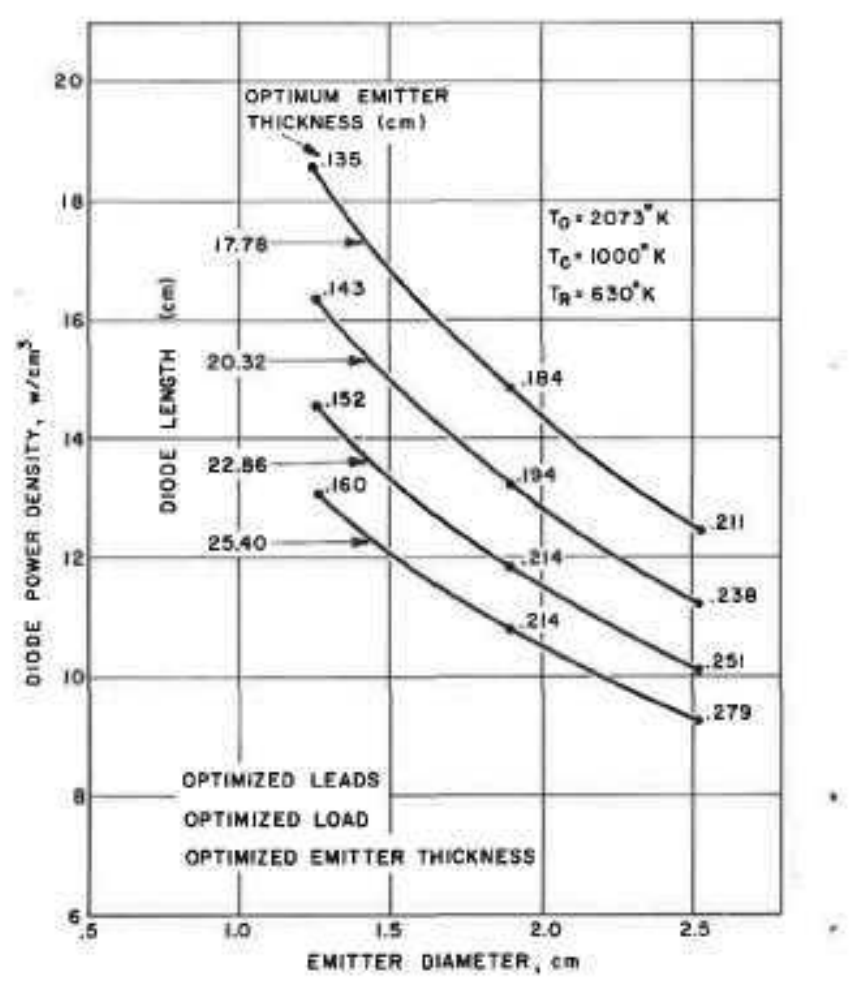


Fig. 5: Axial Profiles with Uniform Fuel

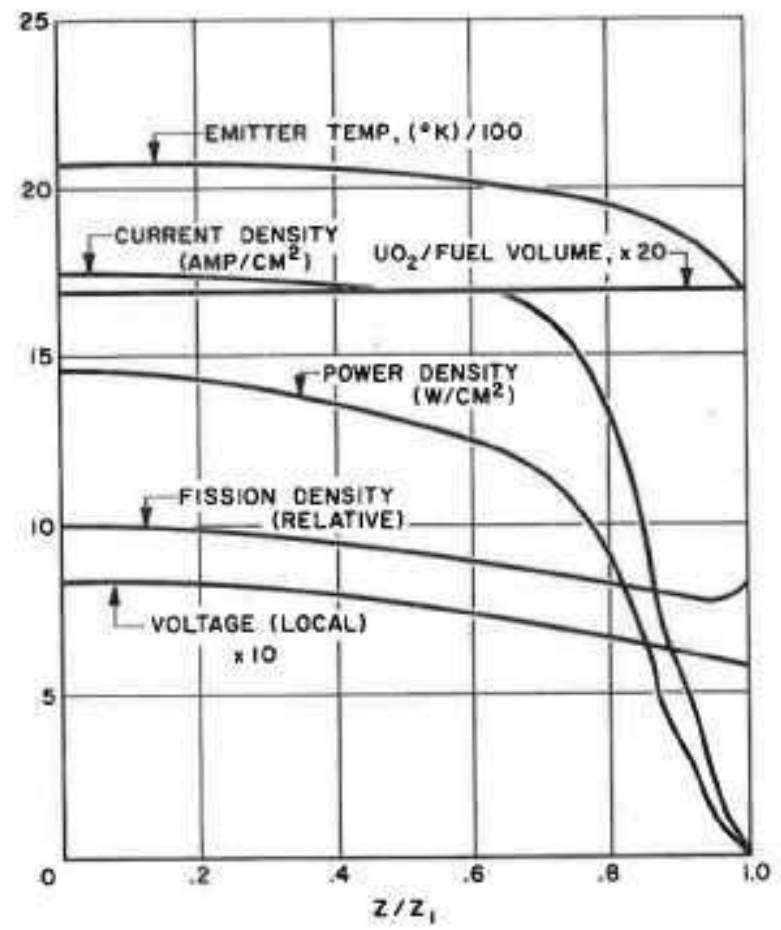

Fig. 7: I-V Characteristics at Constant Heat Input

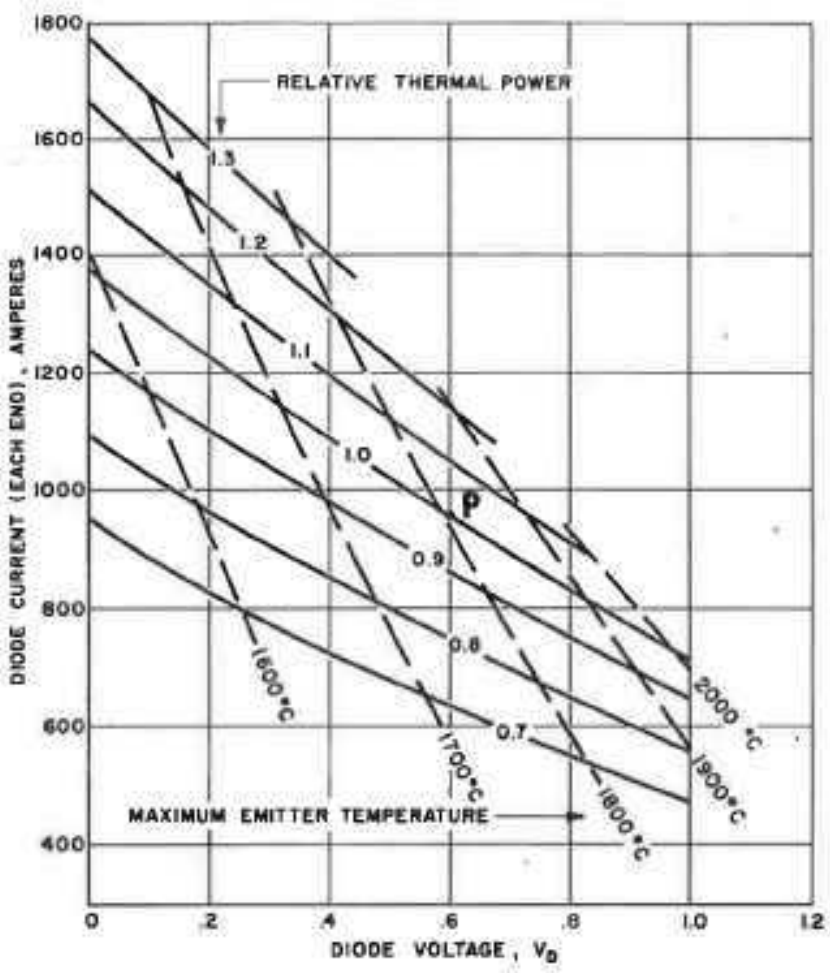

Fig. 6: Axial Profiles with Zoned Fuel

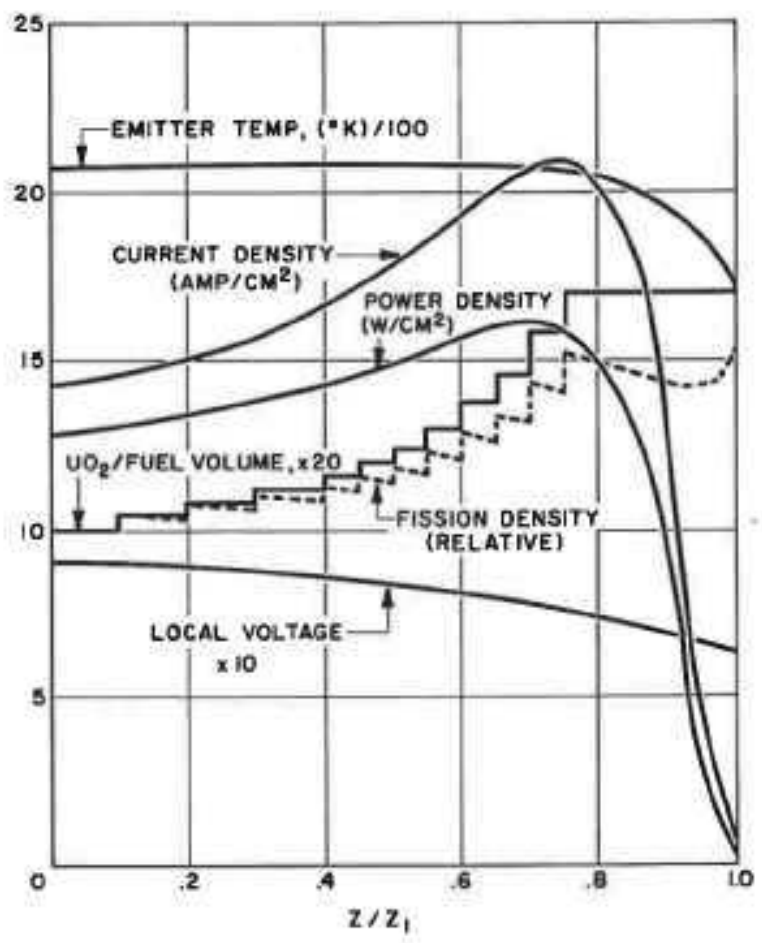

Fig. 8: Example of Dual Solutions

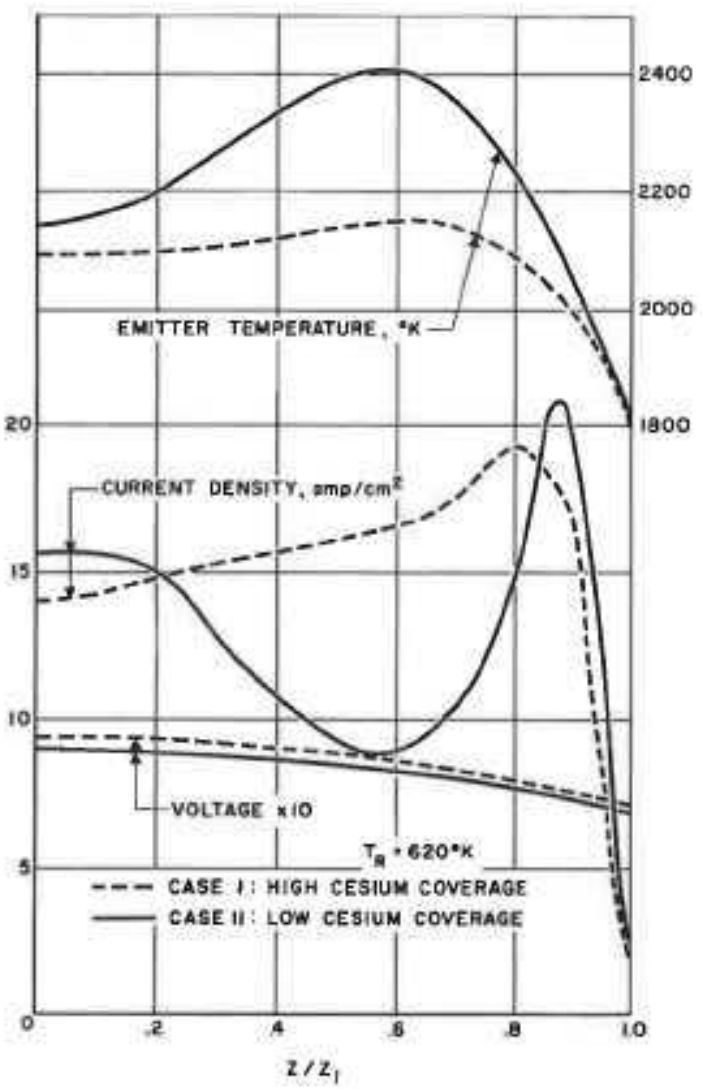

